\title{
Recognition of Off-Line Handwritten Devnagari Characters Using Quadratic Classifier
}

\author{
N. Sharma, U. Pal*, F. Kimura**, and S. Pal \\ Computer Vision and Pattern Recognition Unit, Indian Statistical Institute \\ 203 B T Road, Kolkata-700108, India \\ *umapada@isical.ac.in \\ ** Graduate School of Engineering, Mie University 1577 Kurimamachiya-cho \\ TSU, Mie 514-8507, Japan
}

\begin{abstract}
Recognition of handwritten characters is a challenging task because of the variability involved in the writing styles of different individuals. In this paper we propose a quadratic classifier based scheme for the recognition of offline Devnagari handwritten characters. The features used in the classifier are obtained from the directional chain code information of the contour points of the characters. The bounding box of a character is segmented into blocks and the chain code histogram is computed in each of the blocks. Based on the chain code histogram, here we have used 64 dimensional features for recognition. These chain code features are fed to the quadratic classifier for recognition. From the proposed scheme we obtained $98.86 \%$ and $80.36 \%$ recognition accuracy on Devnagari numerals and characters, respectively. We used fivefold cross-validation technique for result computation.
\end{abstract}

\section{Introduction}

Recognition of handwritten characters has been a popular research area for many years because of its various application potentials. Some of its potential application areas are Postal Automation, Bank cheque processing, automatic data entry, etc. There are many pieces of work towards handwritten recognition of Roman, Japanese, Chinese and Arabic scripts, and various approaches have been proposed by the researchers towards handwritten character recognition [1, 6-11]. Although there are many script and languages in India but not much research is done for the recognition of handwritten Indian characters. In this paper, we propose a system towards the recognition of unconstrained off-line handwritten Devnagari characters.

Many pieces of work have been done towards the recognition of Indian printed characters and at present OCR systems are commercially available for some of the printed Indian scripts [3]. Although several pieces of research work exist on Indian printed characters but only a few attempts have been made towards the recognition of Indian off-line handwritten characters [2]. Among off-line handwritten work of 
Indian scripts, maximum research has been done for Bangla. Systems are available for unconstrained off-line Bangla isolated numerals and characters. Also some systems have been developed for unconstrained Bangla handwritten word recognition for Indian postal recognition [19, 21]. Although first research report on handwritten Devnagari characters was published in 1977 [17] but not much research work is done after that. At present researchers have started to work on handwritten Devnagari characters and few research reports are published recently. Hanmandlu and Murthy [13] proposed a Fuzzy model based recognition of handwritten Hindi numerals and they obtained $92.67 \%$ accuracy. Ramteke et al [14] proposed an isolated Marathi handwritten numeral scheme based on invariant moments. They employed a Gaussian Distribution Function for classification and obtained only $87 \%$ accuracy. Bajaj et al [15] employed three different kinds of features namely, density features, moment features and descriptive component features for classification of Devnagari Numerals. They proposed a multi-classifier connectionist architecture for increasing the recognition reliability and they obtained 89.6\% accuracy. Kumar and Singh [16] proposed a Zernike moment feature based approach for Devnagari handwritten character recognition. They used an artificial neural network for classification. Sethi and Chatterjee [17] proposed a decision tree based approach for recognition of constrained hand printed Devnagari characters using primitive features. Bhattacharaya et al [18] proposed a Multi-Layer Perceptron (MLP) neural network based classification approach for the recognition of Devnagari handwritten numerals and obtained 91.28\% results. They considered a multi-resolution features based on wavelet transform in their proposed system.

In this paper, we propose a scheme for unconstrained off-line handwritten Devnagari numeral and character recognition using quadratic classifier, based on the feature obtained from chain code histogram. Here the bounding box of a character is segmented into blocks and chain code histogram is computed in each blocks. This chain code features are then fed to the classifier for recognition.

Rest of the paper is organized as follows. In Section 2 we discuss about Devnagari language, its character set and the preprocessing of the data used for the proposed scheme. Feature extraction procedure is presented in Section 3. Section 4 details the classifier used for the recognition. The experimental results are discussed in Section 5. Conclusion on the paper is given in Section 6.

\section{Devnagari Language and Data Collection}

Devnagari is the most popular script in India and the most popular Indian language Hindi is written in Devnagari script. Nepali, Sanskrit and Marathi are also written in Devnagari script. Moreover, Hindi is the national language of India and Hindi is the third most popular language in the world [2]. Thus, the work on Devnagari script is very useful for the country. 
The alphabet of the modern Devnagari script consists of 14 vowels and 37 consonants. These characters may be called basic characters. The basic characters of Devnagari script are shown in Fig.1. Writing style in Devnagari script is from left to right. The concept of upper/lower case is absent in Devnagari script. In Devnagari script a vowel following a consonant takes a modified shape. Depending on the vowel, its modified shape is placed at the left, right (or both) or bottom of the consonant. These modified shapes are called modified characters. A consonant or vowel following a consonant sometimes takes a compound orthographic shape, which we call as compound character. Compound characters can be combinations of two consonants as well as a consonant and a vowel. Compounding of three or four characters also exists in these two scripts. There are about 280 compound characters in Devnagari [2].

A Devnagari text line can be partitioned into three zones. The upper-zone denotes the portion above the head-line, the middle zone covers the portion between head-line and base-line, the lower-zone is the portion below base-line.

\begin{tabular}{|c|c|c|c|c|c|c|}
\hline 35 & $2 \pi$ & ¿ & ह & 3 & $r^{2}$ & $x_{3}$ \\
\hline & J & ऐ & ऊो & गी & $3^{\circ}$ & $\overrightarrow{H O}$ \\
\hline
\end{tabular}

(a)

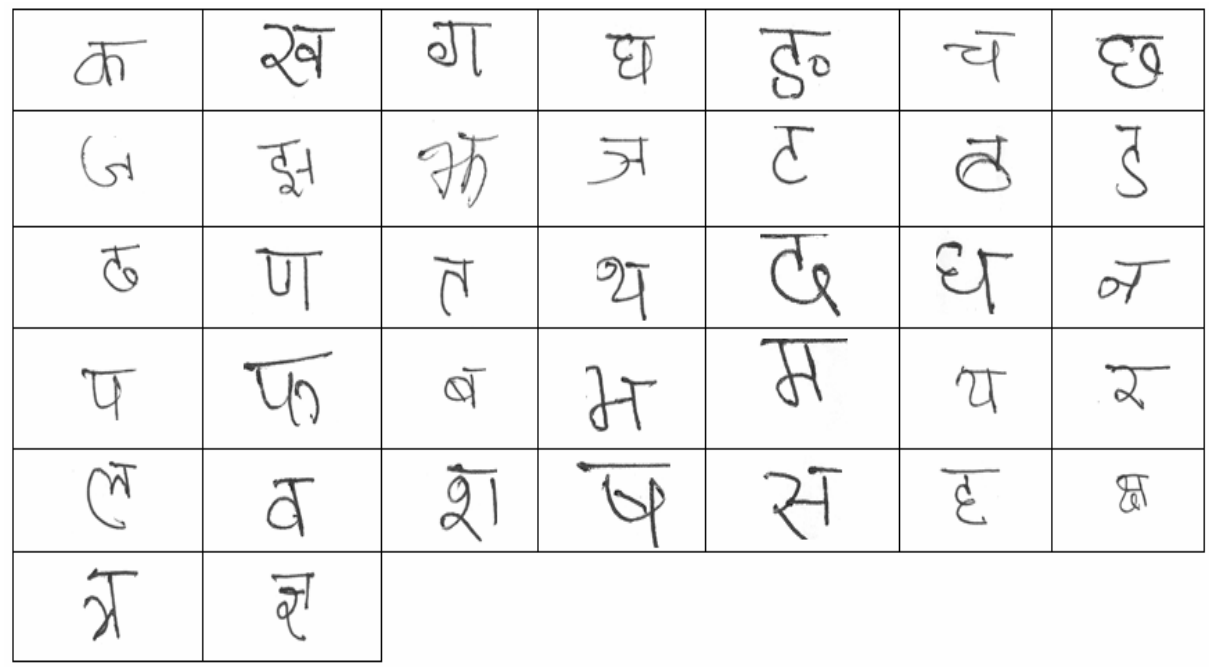

(b)

Fig. 1. Samples of handwritten Devnagari basic characters (a) Vowels, (b) Consonants 
Because of the writing styles of different individuals, characters can have different shapes. As a result recognition of unconstrained handwritten characters becomes a difficult task. To get an idea about such shape difference between printed and handwritten text, printed Devnagari numerals and their coresponding handwritten samples are shown in Table 1.

Table 1. Examples of printed and handwritten Devnagari numerals

\begin{tabular}{|c|c|c|c|c|c|c|}
\hline Numeral & $\begin{array}{c}\text { Printed Devnagari } \\
\text { numeral }\end{array}$ & \multicolumn{5}{|c|}{ Handwritten Devnagari numeral } \\
\hline $\mathbf{0}$ & 0 & 0 & 0 & 0 & 2 & \\
\hline 1 & $?$ & 9 & $q$ & 9 & $q$ & 9 \\
\hline 2 & R & 2 & 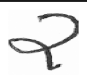 & 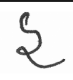 & 2 & 2 \\
\hline 3 & ३ & 3 & 3 & 8 & 3 & 3 \\
\hline 4 & 8 & 8 & 8 & $\gamma$ & 8 & $\delta$ \\
\hline 5 & 4 & $\varphi$ & $y$ & G & 6 & $y$ \\
\hline 6 & $\varepsilon$ & $\xi$ & $\varepsilon_{\rho}$ & $\varepsilon$ & $\varepsilon$ & 8 \\
\hline 7 & $\checkmark$ & 9 & $\sigma$ & (9) & 9 & 9 \\
\hline 8 & $<$ & $C$ & $\tau$ & $U$ & $\zeta$ & $C$ \\
\hline 9 & 9 & 0 & $\rho$ & $S$ & $e$ & $\rho$ \\
\hline
\end{tabular}

In this work we are concerned with the recognition of Devnagari numerals and basic characters only and data used in the present work has been collected from different individuals. For numeral recognition we considered the data discussed in the paper [20]. For the experiment of Devnagari character recognition we collected 11270 samples of vowels and consonants (at least 210 samples of each vowels and consonants). A flat bed scanner was used for digitization. Digitized images are in gray tone with 300 dpi and stored as TIF format. We have used a histogram based global binarizing algorithm to convert them to two-tone ( 0 and 1$)$ images (Here ' 1 'represents object point and '0'represents background point). For removing noises from the images, we have used a method discussed in [3]. 


\section{Feature Extraction}

Histograms of direction chain code of the contour points of the characters are used as feature for recognition [12]. Here we use 64 dimensional features for our recognition purpose. The feature extraction techniques are described below.

\subsection{Dimensional Feature Extraction}

Given a two-tone image, we first find the contour points of the image by the following algorithm. For all object points in the image, consider a 3 x 3 window surrounded to the object point. If any one of the four neighboring points (as shown in Fig.2(a)) is a background point then this object point $(\mathrm{P})$ is considered as contour point. Otherwise it is a non-contour point.

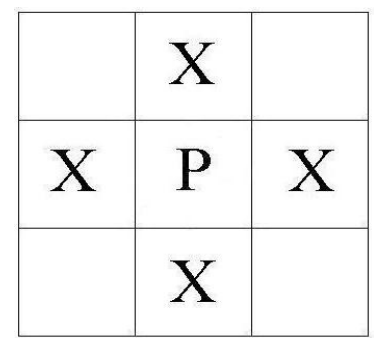

(a)

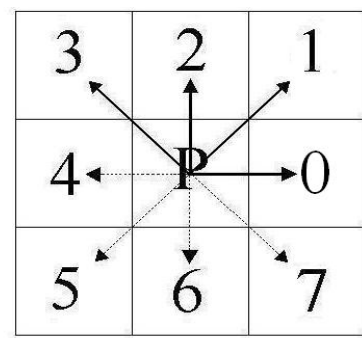

(b)

Fig. 2. (a) For a point $P$ and its four neighbors are shown by ' $X$ ', (b) For a point $P$ the direction codes for its eight neighboring points are shown

The bounding box (minimum rectangle containing the character shown in Fig. 3(b)) of an input character is then divided into 7 x 7 blocks (as shown in Fig.3(c)). In each of these blocks, the direction chain code for each contour point is noted and the frequency of the direction codes is computed. Here we use chain code of four directions only [directions 0 (horizontal), 1 (45 degree slanted), 2(vertical) and 3 (135 degree slanted)]. See Fig.2(b) for illustration of four chain code directions. We assume chain code of direction 0 and 4,1 and 5,2 and 6, 3 and 7, are same. Thus, in each block we get an array of four integer values representing the frequencies and those frequency values are used as feature. Histogram of the values of these four direction codes in each block of a Devnagari numeral is shown in Fig.3(e) . Thus, for $7 \times 7$ blocks we get $7 \times 7 \times 4=196$ features. To reduce the feature dimension, after the histogram calculation in $7 \times 7$ blocks, the blocks are down sampled with a Gaussian filter into 4x4 blocks. As a result we have $4 \times 4 \times 4=64$ features for recognition. Histogram of all the direction obtained after down sampling is shown in Fig.3(f). Example of feature extraction process on a Devnagari character is shown in Fig.4. To normalize the features we compute maximum value of the histograms from all the blocks. We divide each of the above features by this maximum value to get the feature values between 0 and 1 . 


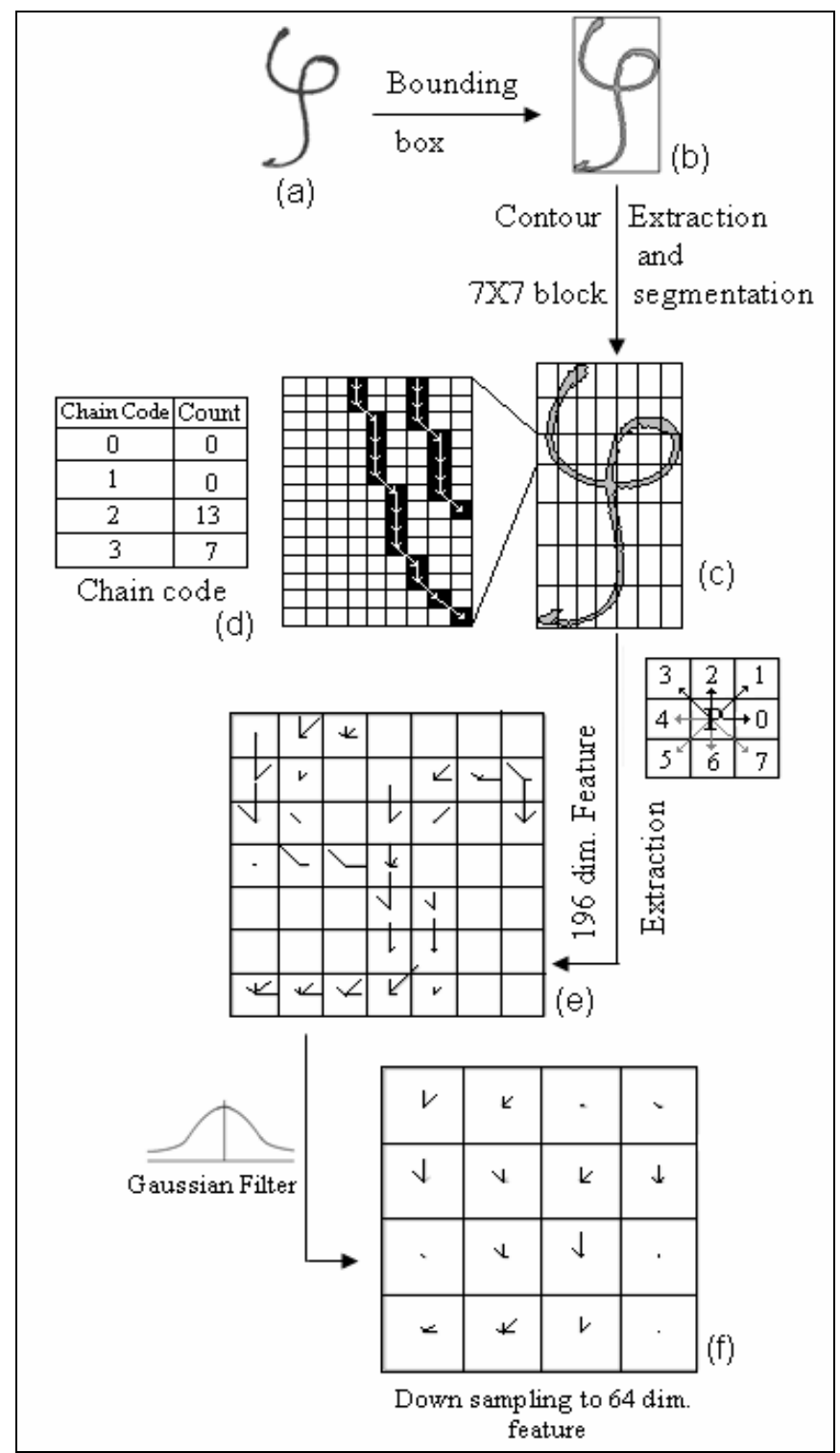

Fig. 3. Pictorial representation of the 64 dimensional feature extraction process for a sample Devnagari numeral. (a) Two tone image of a Devnagari numeral 'five', (b) Bounding box of the numeral. (c) Contour of the numeral shown in black color and the bounding box is segmented into 7 X 7 blocks. (d) Chain code of a block shown in zoomed version. (e) 196 dimensional Chain code features of each block. (f) 64 dimensional features obtained after down sampling using a Gaussian filter. 


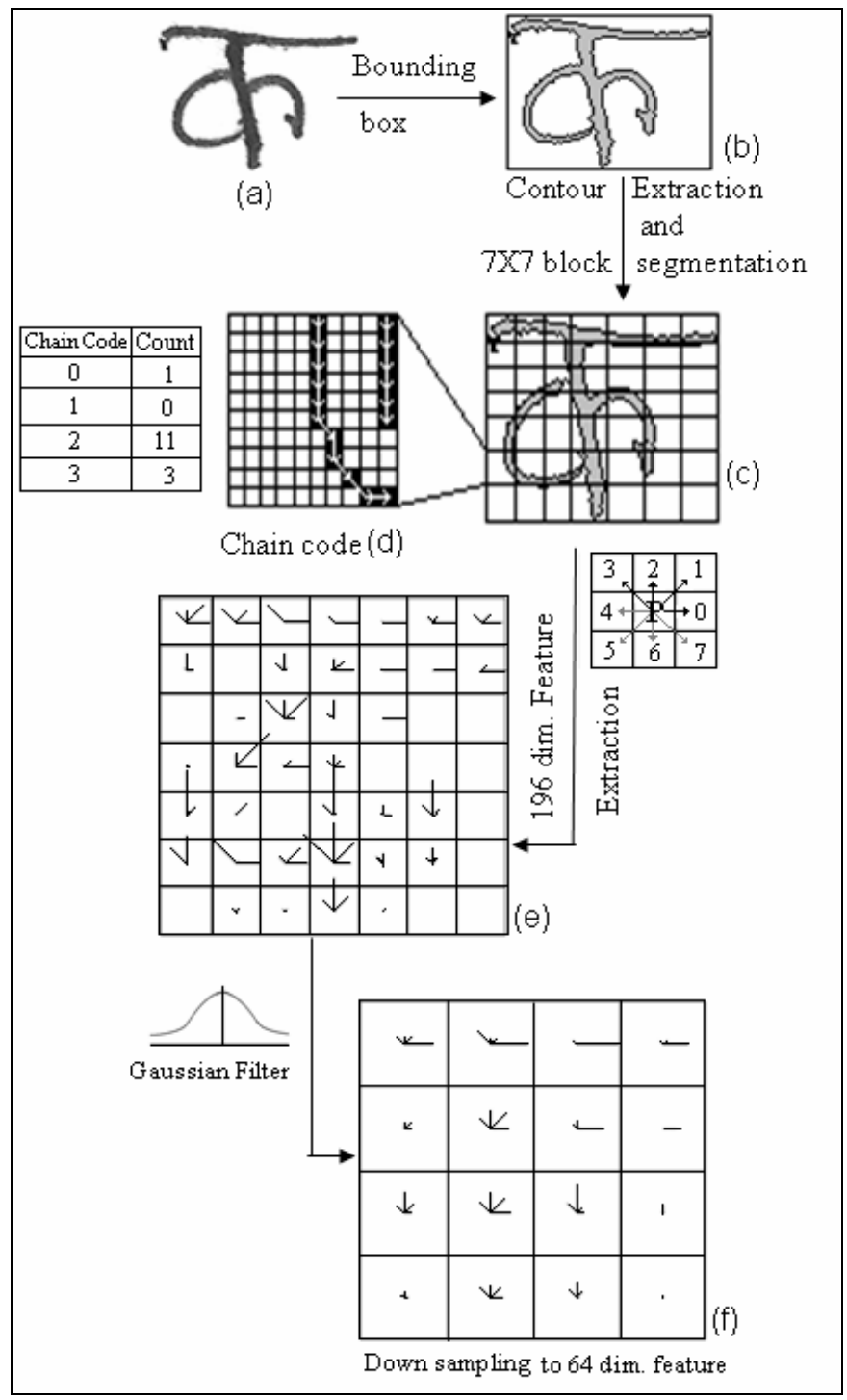

Fig. 4. Pictorial representation of the 64 dimensional feature extraction process for a sample Devnagari character. (a) Two tone image of a Devnagari character, (b) Bounding box of the character. (c) Contour of the character shown in black color and the bounding box is segmented into 7 X 7 blocks. (d) Chain code of a block shown in zoomed version. (e) 196 dimensional Chain code features of each block. (f) 64 dimensional features obtained after down sampling using a Gaussian filter. 


\section{Recognition Classifier}

Many classifiers have been used by the researchers for handwritten character recognition. Here we used a quadratic classifier for our recognition purpose because we noted from the work [12] that this classifier gives better results than other classifiers like Bayes classifier, subspace method etc. Descriptions of the quadratic classifier used for our recognition purpose are given below.

A Modified Quadratic Discriminant function (MQDF) is used by the quadratic classifier [12] which is defined by,

$$
\begin{aligned}
g(X)= & \left(N+N_{0}-n-1\right) \ln \left[1+\frac{1}{N_{o} \sigma^{2}}\left[\|X-M\|^{2}-\sum_{i=1}^{k} \frac{\lambda_{i}}{\lambda_{i}+\frac{N_{o}}{N} \sigma^{2}}\left\{\Phi_{i}^{T}(X-M)\right\}^{2}\right]\right] \\
& +\sum_{i=1}^{k} \ln \left(\lambda_{i}+\frac{N_{o}}{N} \sigma^{2}\right)
\end{aligned}
$$

Where, $X$ is the feature vector of an input character, $M$ is a mean vector of samples, $\phi_{i}^{\mathrm{T}}$ is the $i^{\text {th }}$ eigen vector of the sample covariance matrix, $\lambda_{i}$ is the $i^{\text {th }}$ eigen value of the sample covariance matrix, $n$ is the feature size, $\sigma^{2}$ is the average variance of all classes, $N$ is the average sample of all classes, and $N_{0}$ is selected experimentally and we consider $N_{0}=3 N / 7$ for 64 dimensional feature. We do not use all eigen values and their respective eigen vectors for the classification. We sort the eigen values in descending order and take first 20 eigen values and their respective eigen vectors for classification. Rejection in the system is done if for a character the difference of $1^{\text {st }}$ and $2^{\text {nd }}$ value of $\mathrm{g}(\mathrm{X})$ is smaller than a threshold.

\section{Result and Discussion}

Data used for the present work was collected from different individuals. We digitized 11270 samples of Devnagari characters (vowels as well as consonants) for the experiment of the proposed work. For the experiment of Devnagari numerals we consider the data discussed in [20] and this dataset contains 22,556 handwritten samples.

From experiments we noted that the overall recognition accuracy of the proposed scheme for numerals is $98.86 \%$ and for characters is $80.36 \%$ with zero percent rejection. $99.80 \%$ accuracy was obtained if we consider first two top choices of the recognition results for numerals and $90.56 \%$ accuracy was obtained considering the first two top choices for characters. The detail recognition results with different top choices are given in Table 2. Here we have used five-fold cross validation scheme for recognition result calculation. Here database is divided into 5 subsets and testing is done on each subset using rest of the subsets for learning. The recognition rates for all the subsets are averaged to get accuracy.

From the experiment we noticed that for Devnagari numeral recognition, we can achieve $99.73 \%$ accuracy when we reject $4.25 \%$ of the numeral samples. Also from 
the experiment we noticed that for Devnagari character recognition we obtained $86.41 \%$ accuracy when we reject $7 \%$ samples.

We computed accuracy of the individual Devnagari numerals and their accuracy is given in Table 3. From the experiment we noted the maximum accuracy of $99.81 \%$ was achieved for the Devnagari numeral $\mathcal{O}$ (zero). The next highest accuracy of about $99.66 \%$ was achieved for the numeral $\gamma$ (four). We also noted that the lowest accuracy of $97.15 \%$ was achieved for numeral 2 (three).

The accuracy of individual Devnagari characters was also computed. Maximum accuracy of $90.48 \%$ was achieved for the Devnagari characters 3 and this is because of its unique shape. The next highest accuracy of about $90.13 \%$ was achieved for the character अं.

We also noticed the main confusing pairs of Devnagari characters and their error rates are shown in Table 4. The characters $\mathcal{E}$ and $छ$ confused the most, having an error rate of $0.62 \%$. The next most confusing pair is 2 and $U$, having an error of about $0.35 \%$. From the experiments we noticed that mainly similar shaped characters are confused by the system at higher rate.

Table 2. Recognition results for Devnagari numerals and characters based on different choices from top (without any rejection)

\begin{tabular}{|c|c|c|}
\hline $\begin{array}{c}\text { Top } \\
\text { choices }\end{array}$ & $\begin{array}{c}\text { Accuracy for } \\
\text { Numerals }\end{array}$ & $\begin{array}{c}\text { Accuracy for } \\
\text { Characters }\end{array}$ \\
\hline \hline 1 & $98.86 \%$ & $80.36 \%$ \\
\hline 2 & $99.80 \%$ & $90.56 \%$ \\
\hline 3 & $99.92 \%$ & $94.28 \%$ \\
\hline 4 & $99.96 \%$ & $96.17 \%$ \\
\hline 5 & $99.98 \%$ & $97.77 \%$ \\
\hline
\end{tabular}

Table 3. Recognition results of individual numerals

\begin{tabular}{|c|c||c|c|}
\hline Numeral & Accuracy & Numeral & Accuracy \\
\hline \hline 1 & $99.49 \%$ & 2 & $98.21 \%$ \\
\hline 3 & $97.15 \%$ & 4 & $99.66 \%$ \\
\hline 5 & $98.57 \%$ & 6 & $98.69 \%$ \\
\hline 7 & $98.79 \%$ & 8 & $99.63 \%$ \\
\hline 9 & $98.77 \%$ & 0 & $99.81 \%$ \\
\hline
\end{tabular}


Table 4. Main Confusing pairs among Devnagari characters

\begin{tabular}{|c|c|c|}
\hline Class & Classified as & $\%$ of error \\
\hline$\varepsilon$ & $\bar{घ}$ & $0.62 \%$ \\
\hline 24 & य & $0.35 \%$ \\
\hline$C_{l}$ & तe & $0.34 \%$ \\
\hline $\mathrm{H}$ & H & $0.23 \%$ \\
\hline d & $\Phi$ & $0.22 \%$ \\
\hline
\end{tabular}

We compared our results with the existing pieces of work on off-line handwritten Devnagari numerals and characters. To the best of our knowledge, Hanmandlu and Murthy [13] reports the highest recognition accuracy of about $92.67 \%$ for numerals. But we have obtained an accuracy of about $98.86 \%$, which is $6.19 \%$ better than the results reported by Hanmandlu and Murthy [13]. For Devnagari characters we obtained 80.36\% accuracy using 11270 samples of data, but Kumar and Singh [16] reported $80 \%$ accuracy and they tested only 200 data samples. The details about the comparison results on Devnagari numerals and characters are given in Table 5 and Table 6, respectively.

Table 5. Comparison results for Devnagari Numerals

\begin{tabular}{|c|l|c|}
\hline SI. No. & \multicolumn{1}{|c|}{ Method proposed by } & $\begin{array}{c}\text { Accuracy } \\
\text { obtained }\end{array}$ \\
\hline \hline 1. & Hanmandlu and Ramana Murthy [13] & $92.67 \%$ \\
\hline 2. & Ramteke et al. [14] & $87 \%$ \\
\hline 3. & Bajaj et al. [15] & $89.6 \%$ \\
\hline 4. & Bhattacharaya et al.[18] & $91.28 \%$ \\
\hline 5. & Our proposed method & $\mathbf{9 8 . 8 6 \%}$ \\
\hline
\end{tabular}

Table 6. Comparison results for Devnagari Characters

\begin{tabular}{|c|l|c|c|}
\hline $\begin{array}{c}\text { SI. } \\
\text { No. }\end{array}$ & \multicolumn{1}{|c|}{ Method proposed by } & $\begin{array}{c}\text { Data } \\
\text { size }\end{array}$ & $\begin{array}{c}\text { Accuracy } \\
\text { obtained }\end{array}$ \\
\hline \hline 1. & Kumar and Singh [16] & 200 & $80 \%$ \\
\hline 2. & Proposed method & 11270 & $\mathbf{8 0 . 3 6 \%}$ \\
\hline
\end{tabular}




\section{Conclusion}

India is a multi-lingual and multi-script country comprising of twelve different scripts. But not much work has been done towards off-line handwriting recognition. In this paper we present a quadratic classifier based system for the recognition of unconstrained off-line Devnagari handwritten characters. Dimension of the feature vector was 64, and the features are obtained based on the directional chain codes of the contour of the character. From the experiment we obtained encouraging results. This work will be helpful for the research towards the recognition of other Indian script characters.

\section{References}

1. R. Plamondon and S. N. Srihari, "On-Line and off-line handwritten recognition: A comprehensive survey", IEEE Trans on PAMI, Vol.22, pp.62-84, 2000.

2. U. Pal and B.B. Chaudhuri, "Indian script character recognition: A Survey", Pattern Recognition, Vol. 37, pp. 1887-1899, 2004.

3. B. B. Chaudhuri and U. Pal, "A complete printed Bangla OCR system", Pattern Recognition, vol. 31, pp. 531-549, 1998.

4. K. G. Aparna, A. G. Ramakrishnan: A Complete Tamil Optical Character Recognition System. Proc. in the $5^{\text {th }}$ Intl workshop on Document Analysis and Systems , pp. 53-57, 2002

5. Veena Bansal and R.M.K. Sinha, "Integrating Knowledge Sources in Devanagari Text Recognition”, IEEE Transaction on Systems, Man and Cybernetics, Vol. 30, 4, 2000.

6. D. Yu and H. Yan, "Reconstruction of broken handwritten digits based on structural morphological features", Pattern Recognition, Vol.34, No.2, pp.235-254, 2001.

7. J.Cai and Z.Q.Liu, "Integration of structural and statistical information for unconstrained handwritten character recognition”, IEEE PAMI, Vol. 21, pp.263$270,1999$.

8. H.Byan and S.W. Lee, "A Survey on pattern recognition application of support vector machines”, IJPRAI 17, 459-486, 2003.

9. P. Wunsch and A.F. Laine, "Wavelet Descriptors for Multi-resolution Recognition of Hand-printed Digits", Pattern Recognition, Vol.28, pp.56-66, 1995.

10. Z.Chin and H. Yan, "A handwritten character recognition using self-organizing maps and fuzzy rules”, Pattern Recognition, Vol.22, pp. 923-937, 2000.

11. K.Kim and S.Y. Bang, "A handwritten character classification using tolerant Rough set", IEEE Trans. on PAMI, Vol.22, pp.923-937, 2000.

12. T. Wakabayashi, S. Tsuruoka, F. Kimura and Y. Miyake, "Increasing the Feature size in handwritten Numeral Recognition to improve accuracy, System and Computers in Japan, Vol.26, No.8, pp.35-44, 1995.

13. M. Hanmandlu and O.V. Ramana Murthy, "Fuzzy Model Based Recognition of Handwritten Hindi Numerals", Intl.Conf. on Cognition and Recognition, pp. 490-496, 2005.

14. R.J. Ramteke, P.D.Borkar, S.C. Mehrotra, "Recognition of Marathi Handwritten Numerals: An Invariant Moments Approach", Intl.Conf. on Cognition and Recognition, pp. 482-489, 2005. 
15. Reena Bajaj, Lipika Dey, and S. Chaudhury, "Devnagari numeral recognition by combining decision of multiple connectionist classifiers", Sadhana, Vol.27, part. 1, pp.-5972, 2002.

16. Satish Kumar and Chandan Singh, "A Study of Zernike Moments and its use in Devnagari Handwritten Character Recognition", Intl.Conf. on Cognition and Recognition, pp. 514520, 2005.

17. I.K. Sethi and B. Chatterjee, "Machine Recognition of constrained Hand printed Devnagari”, Pattern Recognition, Vol. 9, pp. 69-75, 1977.

18. U. Bhattacharya, B. B. Chaudhuri, R. Ghosh and M. Ghosh, "On Recognition of Handwritten Devnagari Numerals", In Proc. of the Workshop on Learning Algorithms for Pattern Recognition (in conjunction with the 18th Australian Joint Conference on Artificial Intelligence), Sydney, pp.1-7, 2005.

19. K. Roy, U. Pal and F. Kimura, "Recognition of Handwritten Bangla Characters", In Proc. 2nd International Conference on Machine Intelligence (ICMI), pp.480-485, 2005.

20. U. Bhattacharya and B. B. Chaudhuri, "Databases for research on recognition of handwritten characters of Indian scripts" In Proc. $8^{\text {th }}$ ICDAR, pp.789-793, 2005.

21. U. Pal, K. Roy and F. Kimura, "A Lexicon Driven Method for Unconstrained Bangla Handwritten Word Recognition", In $10^{\text {th }}$ International Workshop on Frontiers in Handwriting Recognition, 2006 (accepted). 